\title{
The energy spectrum of tau leptons induced by the high energy Earth-skimming neutrinos
}

\author{
Jie-Jun Tseng ${ }^{1, *}$, Tsung-Wen $\mathrm{Yeh}^{2, \dagger}$, H. Athar ${ }^{2,3, ~}$, M. \\ A. Huang ${ }^{4, \S}$, Fei-Fain Lee ${ }^{2, \uparrow}$, and Guey-Lin $\operatorname{Lin}^{2, * *}$ \\ 1 Institute of Physics, Academia Sinica, Taipei 115, Taiwan \\ 2 Institute of Physics, National Chiao-Tung University, Hsinchu 300, Taiwan \\ 3 Physics Division, National Center for Theoretical Sciences, Hsinchu 300, Taiwan \\ 4 Department of Physics, National Taiwan University, Taipei 10617, Taiwan
}

(Dated: October 29, 2018)

\begin{abstract}
We present a semi-analytic calculation of the tau-lepton flux emerging from the Earth, induced by the incident high energy neutrinos interacting inside the Earth for $10^{5} \leq E_{\nu} / \mathrm{GeV} \leq 10^{10}$. We obtain results for the energy dependence of the tau-lepton flux coming from the Earth-skimming neutrinos, because of the neutrino-nucleon charged-current scattering as well as the resonant $\bar{\nu}_{e} e^{-}$ scattering. We illustrate our results for several anticipated high energy astrophysical neutrino sources such as the AGNs, the GRBs, and the GZK neutrino fluxes. The tau lepton fluxes resulting from rock-skimming and ocean-skimming neutrinos are compared. Such comparisons can render useful information for the spectral indices of incident neutrino fluxes.
\end{abstract}

PACS numbers: 95.85.Ry, 14.60.Fg, 14.60.Pq, 95.55.Vj

\footnotetext{
* E-mail: gen@phys.sinica.edu.tw

$\dagger$ E-mail: twyeh@cc.nctu.edu.tw

¥ E-mail: athar@phys.cts.nthu.edu.tw

$\S$ E-mail: huangmh@phys.ntu.edu.tw

ฯ E-mail: u8727515@cc.nctu.edu.tw

** E-mail: glin@cc.nctu.edu.tw
} 


\section{INTRODUCTION}

The detection of high energy neutrinos $\left(E_{\nu}>10^{5} \mathrm{GeV}\right)$ is crucial to identify the extreme energy sources in the Universe, and possibly to unveil the puzzle of cosmic rays with energy above the GZK cutoff[1]. These proposed scientific aims are well beyond the scope of the conventional high energy gamma-ray astronomy. Because of the expected small flux of the high energy neutrinos, large scale detectors $\left(\geq 1 \mathrm{~km}^{2}\right)$ seem to be needed to obtain the first evidence.

There are two different strategies to detect the footprints of high energy neutrinos. The first strategy is implemented by installing detectors in a large volume of ice or water where most of the scatterings between the candidate neutrinos and nucleons occur essentially inside the detector, whereas the second strategy aims at detecting the air showers caused by the charged leptons produced by the neutrino-nucleon scatterings taking place inside the Earth or in the air, far away from the instrumented volume of the detector. The latter strategy thus include the possibility of detection of quasi horizontal incident neutrinos which are also referred to as the Earth-skimming neutrinos. These neutrinos are considered to interact below the horizon of an Earth based surface detector.

The second strategy is proposed only recently 2]. The Pierre Auger observatory group has simulated the anticipated detection of the air-showers from the decays of $\tau$ leptons 3 ]. The tau air-shower event rates resulting from the Earth-skimming tau neutrinos for different high energy neutrino telescopes are given in [4]. A Monte-Carlo study of tau air-shower event rate was also reported not long ago [5]. We note that Ref.[4] does not consider the tau-lepton energy distribution in the $\nu_{\tau}$-nucleon scattering, and only the incident tau neutrinos with energies greater than $10^{8} \mathrm{GeV}$ are considered. For Ref. [5], we note that only the sum of tau air-shower event rates arising from different directions is given. Hence some of the events may be due to tau-leptons/neutrinos traversing a large distance. As a result, it is not possible to identify the source of tau-neutrino flux even with the observation of tau-lepton induced air-shower.

In this work, we shall focus on the high energy Earth-skimming neutrinos and shall calculate the energy spectrum of their induced tau-leptons, taking into account the inelasticity of neutrino-nucleon scatterings and the tau-lepton energy loss in detail. Our work differs

from Ref. 5] by our emphasis on the Earth-skimming neutrinos. We shall present our results 
in the form of outgoing tau-lepton spectra for different distances inside the rock, instead of integrating the energy spectra. As will be demonstrated, such spectra are insensitive to the distances traversed by the Earth-skimming $\nu_{\tau}$ and $\tau$. They are essentially determined by the tau lepton range. Because of this characteristic feature, our results are useful for setting up simulations with specifically chosen air-shower content detection strategy, such as detection of the Cherenkov radiation or the air fluorescence.

We start with our semi-analytic description in Section II. The transport equations governing the evolutions of neutrino and tau-lepton fluxes will be derived. Using these, we then calculate the tau-lepton flux resulting from the resonant $\bar{\nu}_{e} e^{-} \rightarrow W^{-} \rightarrow \bar{\nu}_{\tau} \tau^{-}$scattering. In Section III, we summarize our main results, namely the tau-lepton energy spectra due to neutrino-nucleon scatterings. The implications of our results will be discussed here also. In particular, we shall point out that the ratio of tau-lepton flux induced by rock-skimming neutrinos to that induced by ocean-skimming neutrinos is sensitive to the spectral index of the incident tau-neutrino flux. In Section IV, we discuss some prospects for possible future observations of the associated radiation from these tau leptons.

\section{TAU LEPTON ENERGY SPECTRUM}

Let us begin with the transport equations for tau neutrinos and tau leptons. Considering only the neutrino-nucleon scatterings, we have

$$
\frac{\partial F_{\nu_{\tau}}(E, X)}{\partial X}=-\frac{F_{\nu_{\tau}}(E, X)}{\lambda_{\nu_{\tau}}(E)}+n_{N} \sum_{i=1}^{3} \int_{y_{\min }^{i}}^{y_{\max }^{i}} \frac{\mathrm{d} y}{1-y} F_{i}\left(E_{y}, X\right) \frac{\mathrm{d} \sigma_{\nu}^{i}}{\mathrm{~d} y}\left(y, E_{y}\right),
$$

and

$$
\begin{aligned}
\frac{\partial F_{\tau}(E, X)}{\partial X} & =-\frac{F_{\tau}(E, X)}{\lambda_{\tau}^{\mathrm{cc}}(E)}-\frac{F_{\tau}(E, X)}{\rho d_{\tau}(E)}+\frac{\partial\left[(\alpha(E)+\beta(E) E) F_{\tau}(E, X)\right]}{\partial E} \\
& +n_{N} \int_{y_{\min }}^{y_{\max }} \frac{\mathrm{d} y}{1-y} F_{\nu_{\tau}}\left(E_{y}, X\right) \frac{\mathrm{d} \sigma_{\nu_{\tau} N \rightarrow \tau Y}}{\mathrm{~d} y}\left(y, E_{y}\right),
\end{aligned}
$$

where $n_{N}$ the number of target nucleons per unit medium mass, and $\rho$ is the mass density of the medium. The $\sigma_{\nu}^{1,2,3}$ are defined as $\sigma\left(\nu_{\tau}+N \rightarrow \nu_{\tau}+Y\right), \Gamma\left(\tau \rightarrow \nu_{\tau}+Y\right) / c \rho n_{N}$, and $\sigma\left(\tau+N \rightarrow \nu_{\tau}+Y\right)$ respectively. The quantity $X$ represents the slant depth traversed by the particles, i.e., the amount of medium per unit area traversed by the particle (and thus in units of $\left.\mathrm{g} / \mathrm{cm}^{2}\right)$. The $\lambda_{\nu}, d_{\tau}$, and $\lambda_{\tau}^{\mathrm{CC}}$ represent the $\nu_{\tau}$ interaction thickness, the taulepton decay length, and the tau-lepton charged-current interaction thickness respectively, 
with, say, $\lambda_{\nu}^{-1}=n_{N} \sigma_{\nu N}$ and $d_{\tau}=c \tau_{\tau} E / m_{\tau}$. The $E_{y}$ is equal to $E /(1-y)$, where $y$ is the inelasticity of neutrino-nucleon scatterings, such that the initial and final-state particle energies in the differential cross sections $\mathrm{d} \sigma_{\nu}^{i}\left(y, E_{y}\right) / \mathrm{d} y$ and $\mathrm{d} \sigma_{\nu_{\tau} N \rightarrow \tau Y}\left(y, E_{y}\right) / \mathrm{d} y$ are $E /(1-y)$ and $E$ respectively. The limits for $y, y_{\min }^{i}$ and $y_{\max }^{i}$ depend on the kinematics of each process. Finally, the energy-loss coefficients $\alpha(E)$ and $\beta(E)$ are defined by $-\mathrm{d} E / \mathrm{d} X=\alpha(E)+\beta(E) E$ with $E$ the energy being the tau lepton. An equation similar to Eq. (2) in the context of atmospheric muons was found in Ref. 6 ].

As mentioned before, Eqs. (11) and (2) only take into account neutrino-nucleon scatterings. It is of interest to calculate the tau-lepton fluxes produced by the Glashow resonance [7, 8], namely via $\bar{\nu}_{e} e^{-} \rightarrow W \rightarrow \bar{\nu}_{\tau} \tau^{-}$also. The transport equation for $\bar{\nu}_{e}$ then reads:

$$
\frac{\partial F_{\bar{\nu}_{e}}(E, X)}{\partial X}=-\frac{F_{\bar{\nu}_{e}}(E, X)}{\lambda_{\bar{\nu}_{e}}(E)}+n_{N} \int_{y_{\min }}^{y_{\max }} \frac{\mathrm{d} y}{1-y} F_{\bar{\nu}_{e}}\left(E_{y}, X\right) \frac{\mathrm{d} \sigma_{\bar{\nu}_{e} N \rightarrow \bar{\nu}_{e} Y}}{\mathrm{~d} y}\left(y, E_{y}\right) .
$$

Similarly, the corresponding equation for the tau-lepton flux is given by

$$
\begin{aligned}
\frac{\partial F_{\tau}(E, X)}{\partial X} & =-\frac{F_{\tau}(E, X)}{\lambda_{\tau}^{\mathrm{cc}}(E)}-\frac{F_{\tau}(E, X)}{\rho d_{\tau}(E)} \\
& +n_{e} \int_{y_{\min }}^{y_{\max }} \frac{\mathrm{d} y}{1-y} F_{\bar{\nu}_{e}}\left(E_{y}, X\right) \frac{\mathrm{d} \sigma_{\bar{\nu}_{e} e^{-} \rightarrow \bar{\nu}_{\tau} \tau^{-}}}{\mathrm{d} y}\left(y, E_{y}\right)
\end{aligned}
$$

where $n_{e}$ is the number of target electrons per unit medium mass.

Before solving the above coupled transport equations, it is essential to know the energyloss coefficients $\alpha(E)$ and $\beta(E)$. As pointed out before 9$]$, the coefficient $\alpha(E)$ is due to the energy loss by the ionization[10], while $\beta(E)$ is contributed by the bremsstrahlung[11], the $e^{+} e^{-}$pair production [12] and the photo-nuclear processes [9, 13]. It is understood that the contribution by $\alpha(E)$ becomes unimportant for $E \geq 10^{5} \mathrm{GeV}$. The coefficient $\beta(E)$ can be parameterized as $\beta(E)=\left(1.6+6\left(E / 10^{9} \mathrm{GeV}\right)^{0.2}\right) \times 10^{-7} \mathrm{~g}^{-1} \mathrm{~cm}^{2}$ in the standard rock for $10^{5} \leq E / \mathrm{GeV} \leq 10^{12}$.

It is of interest to check the tau-lepton range given by our semi-analytic approach. To do this, we rewrite Eq. (2) by dropping the neutrino term, i.e.,

$$
\frac{\partial F_{\tau}(E, X)}{\partial X}=-\frac{F_{\tau}(E, X)}{\lambda_{\tau}^{\mathrm{cc}}(E)}-\frac{F_{\tau}(E, X)}{\rho d_{\tau}(E)}+\frac{\partial\left[\gamma(E) F_{\tau}(E, X)\right]}{\partial E},
$$

with $\gamma(E) \equiv \alpha(E)+\beta(E) E$. One can easily solve it for $F_{\tau}(E, X)$ :

$$
F_{\tau}(E, X)=F_{\tau}(\bar{E}, 0) \exp \left[\int_{0}^{X} \mathrm{~d} T\left(\gamma^{\prime}(\bar{E})-\frac{1}{\rho d_{\tau}(\bar{E})}-\frac{1}{\lambda_{\tau}^{\mathrm{cc}}(\bar{E})}\right)\right]
$$


where $\bar{E} \equiv \bar{E}(X ; E)$ with $\mathrm{d} \bar{E} / \mathrm{d} X=\gamma(\bar{E})$ and $\bar{E}(0 ; E)=E$. To calculate the tau-lepton range, we substitute $F_{\tau}(E, 0)=\delta\left(E-E_{0}\right)$. The survival probability $P\left(E_{0}, X\right)$ for a taulepton with an initial energy $E_{0}$ at $X=0$ is

$$
P\left(E_{0}, X\right)=\frac{\gamma\left(\tilde{E}_{0}\right)}{\gamma\left(E_{0}\right)} \exp \left[\int_{0}^{X} \mathrm{~d} T\left(\gamma^{\prime}\left(\tilde{E}_{0}\right)-\frac{1}{\rho d_{\tau}\left(\tilde{E}_{0}\right)}-\frac{1}{\lambda_{\tau}^{\mathrm{cc}}\left(\tilde{E}_{0}\right)}\right)\right],
$$

where $\tilde{E}_{0} \equiv \tilde{E}\left(X ; E_{0}\right)$ with $\mathrm{d} \tilde{E}_{0} / \mathrm{d} X=-\gamma\left(\tilde{E}_{0}\right)$ and $\tilde{E}_{0}\left(0 ; E_{0}\right)=E_{0}$. The tau-lepton range is simply

$$
R_{\tau}\left(E_{0}\right)=\int_{0}^{\infty} \mathrm{d} X P\left(E_{0}, X\right)
$$

For $E_{0}=10^{9} \mathrm{GeV}$, we find that $R_{\tau}=10.8 \mathrm{~km}$ in the standard rock $(Z=11, A=22)$ while $R_{\tau}=5.0 \mathrm{~km}$ in the iron. Both values are in good agreement with those obtained by Monte-Carlo calculations [9]. To compare the tau-lepton ranges, we have followed the convention in Ref. 9] by requiring the final tau-lepton energy $\tilde{E}\left(X ; E_{0}\right)$ to be greater than $50 \mathrm{GeV}$.

It is to be noted that we obtain $R_{\tau}$ by using the continuous tau-lepton energy-loss approach, rather than stochastic approach adopted in Ref.[9]. In the muon case, the continuous approach to the muon energy loss is known to overestimate the muon range 14$]$. Such an overestimate is not significant in the tau-lepton case, because of the decay term in Eq. (7). In fact, tau lepton decay term dictates the tau range in the rock until $E_{\tau} \geq 10^{7}$ $\mathrm{GeV}$. Even for $E_{\tau}>10^{7} \mathrm{GeV}$, the tau lepton range is still not entirely determined by the tau-lepton energy loss. Hence different treatments on the tau-lepton energy loss do not lead to large differences in the tau-lepton range, in contrast to the case for the muon range. Our result for the tau-lepton range up to $10^{12} \mathrm{GeV}$ are plotted in Fig. 1] This is an extension of the result in Ref.[9], where the tau-lepton range is calculated only up to $10^{9} \mathrm{GeV}$. Our extension is seen explicitly in the addition of charged-current scattering term on the R.H.S. of Eq. (5). This term is necessary because $1 / \lambda_{\tau}^{\mathrm{CC}}$ becomes comparable to $1 / \rho d_{\tau}$ in the rock for $E \geq 10^{10} \mathrm{GeV}$; whereas one does not need to include the contribution by the tau-lepton neutral-current scattering, since such a contribution can not compete with the last term in Eq. (5]) until $E \geq 10^{16} \mathrm{GeV}[9]$. We remark that our extended results for $R_{\tau}$ are subject to the uncertainties of neutrino-nucleon scattering cross section at high energies. We use the CTEQ6 parton distribution functions [15] in this work, and at the high energy (the small $x$ region, namely for $x<10^{-6}$ ), we fit these parton distribution functions into the form proportional to $x^{-1.3}$ as a guide. 
Having checked the tau-lepton range, we now proceed to calculate the tau-lepton flux. It is instructive to begin with the simple case: the $\bar{\nu}_{e} e^{-}$resonant scattering. It is well known that [7, 8]

$$
\sigma\left(\bar{\nu}_{e} e^{-} \rightarrow W^{-} \rightarrow \bar{\nu}_{\tau} \tau^{-}\right)=\frac{G_{F}^{2} m_{W}^{4}}{3 \pi} \cdot \frac{s}{\left(s-m_{W}^{2}\right)^{2}+m_{W}^{2} \Gamma_{W}^{2}},
$$

with $s=2 m_{e} E_{\overline{\nu_{e}}}$ and $1 / \sigma \cdot \mathrm{d} \sigma / \mathrm{d} z=3(1-z)^{2}$, where $z=E_{\tau} / E_{\bar{\nu}_{e}}$. We shall only focus on those $\bar{\nu}_{e}$ 's for which $E_{\bar{\nu}_{e}}$ satisfies the resonance condition, i.e., $E_{\bar{\nu}_{e}} \approx E_{R} \equiv m_{W}^{2} / 2 m_{e}$. It is clear from Eq. (41) that $F_{\tau}(E, X)$ only depends on $F_{\bar{\nu}_{e}}\left(E_{R}, X\right)$, because of the narrow peak nature of $\bar{\nu}_{e} e^{-}$scattering cross section. One also expects that $F_{\tau}(E, X)$ is only significant for $E$ around the resonance energy $E_{R}$. In this energy region, one may neglect the first term on the R.H.S. of Eq. (44) in comparison with the second term. In the narrow width approximation, the last term in Eq. (4) can be recast into $\frac{1}{3}\left(1-E / E_{R}\right)^{2}\left(\pi \Gamma_{W} / L_{R} m_{W}\right) F_{\bar{\nu}_{e}}\left(E_{R}, X\right)$, where $\Gamma_{W}$ is the width of the $W$ boson while $L_{R}$ is the interaction thickness of the resonant $\bar{\nu}_{e} e^{-} \rightarrow W^{-}$ scattering (see Appendix A for details). The tau lepton flux can be readily obtained once $F_{\bar{\nu}_{e}}\left(E_{R}, X\right)$ is given. We observe that the regeneration term in Eq. (30) (second term on the R.H.S.) can be neglected as it is necessarily off the $W$ boson peak. Hence, we easily obtain $F_{\bar{\nu}_{e}}\left(E_{R}, X\right)=\exp \left(-X / L_{R}\right) F_{\bar{\nu}_{e}}\left(E_{R}, 0\right)$. Substituting this expression into Eq. (4), we obtain

$$
\frac{F_{\tau}(E, X)}{F_{\bar{\nu}_{e}}\left(E_{R}, 0\right)}=3.3 \cdot 10^{-4} \times\left(\frac{E}{E_{R}}\right) \times\left(1-\frac{E}{E_{R}}\right)^{2} \times \exp \left(-\frac{X}{L_{R}}\right),
$$

in the limit $X \gg \rho d_{\tau}$. The pre-factor $3.3 \cdot 10^{-4}$ is obtained by assuming a standard-rock medium. In water it becomes $1.4 \cdot 10^{-4}$. It is to be noted that $E<E_{R}$ in the above equation. We shall see later that the contribution to $F_{\tau}(E, X)$ by the $W$-resonance is negligible compared to that by the $\nu_{\tau}-N$ scattering.

Let us now turn to the case of tau-lepton production by the $\nu_{\tau}-N$ charged-current scattering. The tau-lepton flux can be calculated from Eqs. (11) and (2) once the incoming $\nu_{\tau}$ flux is given. The $\nu_{\tau}$ flux can be obtained by the following ansatz[16]:

$$
F_{\nu_{\tau}}(E, X)=F_{\nu_{\tau}}(E, 0) \exp \left(-\frac{X}{\Lambda_{\nu}(E, X)}\right),
$$

where $\Lambda_{\nu}(E, X)=\lambda_{\nu}(E) /\left(1-Z_{\nu}(E, X)\right)$, with the factor $Z_{\nu}(E, X)$ arising from the regeneration effect of the $\nu_{\tau}$ flux. On the other hand, the tau-lepton flux is given by

$$
\begin{aligned}
F_{\tau}(E, X) & =\int_{0}^{X} \mathrm{~d} T G_{\nu}(\bar{E}, T) \\
& \times \exp \left[\int_{T}^{X} \mathrm{~d} T^{\prime}\left(\gamma^{\prime}(\bar{E})-\frac{1}{\rho d_{\tau}(\bar{E})}-\frac{1}{\lambda_{\tau}^{\mathrm{cc}}(\bar{E})}\right)\right],
\end{aligned}
$$


with $\bar{E} \equiv \bar{E}(X-T ; E)$, and

$$
G_{\nu}(E, X)=n_{N} \int_{y_{\min }}^{y_{\max }} \frac{\mathrm{d} y}{1-y} F_{\nu}\left(E_{y}, X\right) \frac{\mathrm{d} \sigma_{\nu_{\tau} N \rightarrow \tau Y}}{\mathrm{~d} y}\left(y, E_{y}\right) .
$$

It is easy to see that the factor $Z_{\nu}(E, X)$ enters into the expression for $F_{\tau}(E, X)$ through the function $G_{\nu}(E, X)$. Similarly, $Z_{\nu}(E, X)$ also depends on $F_{\tau}(E, X)$. It is possible to solve for $Z_{\nu}(E, X)$ and $F_{\tau}(E, X)$ simultaneously by the iteration method[16]. The details are given in Appendix B.

\section{RESULTS AND DISCUSSION}

In the following, we show the tau-lepton fluxes resulting from three kinds of diffuse astrophysical neutrino fluxes: the AGN [17], GRB 18] and GZK[19] neutrino fluxes. In these representative models, $F_{\nu_{\tau}}$ arises because of neutrino flavor mixing [20]. The $p \gamma$ interactions are the source of intrinsic $F_{\nu_{\mu}}$, and $F_{\nu_{\tau}}=1 / 2 \cdot F_{\nu_{\mu}}$ because of (two) neutrino flavor oscillations during propagation. Our convention for $F_{\nu_{\tau}}$ is that $F_{\nu_{\tau}}=\mathrm{d} N_{\nu_{\tau}} / \mathrm{d}\left(\log _{10} E\right)$ in the unit of $\mathrm{cm}^{-2} \mathrm{~s}^{-1} \mathrm{sr}^{-1}$. The same convention is used for the outgoing tau lepton fluxes.

In Fig. 2, we show the outgoing tau-lepton energy spectra resulting from the propagation of incident AGN neutrinos inside the rock $\left(\rho=2.65 \mathrm{~g} / \mathrm{cm}^{3}\right)$ for $X / \rho=10 \mathrm{~km}, 100 \mathrm{~km}$ and $500 \mathrm{~km}$ respectively. It is interesting to see that the tau-lepton energy spectra remain almost unchanged for the above three different slant depths/matter density ratio values. This feature can be understood by two simple facts. First of all, the neutrino-nucleon chargedcurrent interaction length, which is related to the interaction thickness by $\lambda_{\mathrm{CC}}=\rho l_{\mathrm{CC}}$, is

given by $l_{\mathrm{CC}}=2 \cdot 10^{4} \mathrm{~km}\left(\frac{1 \mathrm{~g} / \mathrm{cm}^{3}}{\rho}\right)\left(\frac{E_{\nu}}{10^{6} \mathrm{GeV}}\right)^{-0.363}$. Secondly, the tau leptons, which eventually exit the Earth, ought to be produced within a tau-lepton-range distance to the exit point. For a tau-lepton produced far away from the exit point, it loses energy and decays before reaching to the exit point. Hence the tau-lepton flux is primarily determined by the ratio of tau-lepton range to the charged current neutrino-nucleon interaction length. The total slant depth $X$ which the tau-neutrino (tau-lepton) traverses inside the Earth is then unimportant, unless $X$ is large enough such that the tau neutrino flux attenuates significantly before tau-neutrino is converted into the tau-lepton. We note that the typical energy for the AGN neutrinos, in which this flux peaks, is between $10^{5}$ and $10^{8} \mathrm{GeV}$. The corresponding neutrino-nucleon neutral current interaction length then ranges from 42, $000 \mathrm{~km}$ down to 
$3,400 \mathrm{~km}$, given $l_{\mathrm{NC}}=2.35 \cdot l_{\mathrm{CC}}$. Hence, even for $X / \rho$ as large as $500 \mathrm{~km}$, the attenuation of the tau neutrino flux is negligible. This explains the insensitivity of tau-lepton flux with respect to our chosen $X / \rho$ values for the AGN case. The situation is rather similar for the tau-lepton flux resulting from the GRB tau neutrinos (see Fig. 3). On the other hand, a slight suppression is found for the GZK case at $E_{\tau}>10^{9} \mathrm{GeV}$ as one increases $X / \rho$ from $10 \mathrm{~km}$ to $500 \mathrm{~km}$ (see Fig. 4). This is because the typical GZK tau neutrino flux peaks for energy range between $10^{7}$ and $10^{10} \mathrm{GeV}$, which corresponds to attenuation lengths ranging from 7, $800 \mathrm{~km}$ down to $640 \mathrm{~km}$. One notices that $640 \mathrm{~km}$ is rather close to the distance 500 $\mathrm{km}$ which we choose for $X / \rho$. Hence a slight suppression on the tau-lepton flux occurs for $X / \rho=500 \mathrm{~km}$.

We have compared our AGN-type tau-lepton flux with that obtained by Monte-Carlo simulations, adopting stochastic approach for the tau-lepton energy loss [21]. The two taulepton fluxes agree within $\sim 10 \%$. This is expected since the tau-lepton range obtained by the above two approaches agree well, as pointed out before. It is easily seen from Fig. 2 to Fig. 4 that the AGN case has a largest tau-lepton flux between $10^{6}$ and $10^{8} \mathrm{GeV}$. Since the resonant $\bar{\nu}_{e}-e^{-}$scattering cross section peaks at $E_{\nu}=6.3 \cdot 10^{6} \mathrm{GeV}$, it is of interest to compare the integrated tau-lepton flux resulting from this scattering to the one arising from neutrino-nucleon scattering. For the former case, we integrate the tau-lepton energy spectrum from $10^{6} \mathrm{GeV}$ to $6.3 \cdot 10^{6} \mathrm{GeV}$, and obtain $\Phi_{\nu}^{\mathrm{R}}=0.08 \mathrm{~km}^{-2} \mathrm{sr}^{-1} \mathrm{yr}^{-1}$. For neutrino-nucleon scattering, we find that $\Phi_{\nu}^{\mathrm{CC}}=2.2 \mathrm{~km}^{-2} \mathrm{sr}^{-1} \mathrm{yr}^{-1}$ by integrating the corresponding tau-lepton energy spectrum from $10^{6} \mathrm{GeV}$ to $10^{7} \mathrm{GeV}$. The detailed results for $\Phi_{\nu}^{\mathrm{CC}}$ are summarized in Table 1 . The entries in the Table entitled full are obtained using the $F_{\tau}$ obtained in this work, whereas the approximated values entitled approx are obtained by following the description given in Ref. [4], which uses a constant $\beta$ and a constant inelasticity coefficient for $\nu_{\tau} N$ scattering. We remark that the authors in Ref. [4] have taken $E$ to be greater than $10^{8} \mathrm{GeV}$. Hence the integrated fluxes in the column approx with energies less than $10^{8} \mathrm{GeV}$ are taken as extrapolations. Thus, one should compare the two integrated fluxes only for $E>10^{8} \mathrm{GeV}$. One can see that the two integrated fluxes seem to agree for $E>10^{8} \mathrm{GeV}$. Besides the integrated fluxes for $E>10^{8} \mathrm{GeV}$, we also obtain integrated tau-lepton fluxes for $10^{6} \leq E / \mathrm{GeV} \leq 10^{8}$. It is easily seen that, in this energy range, the integrated tau-lepton flux from Earth-skimming AGN neutrinos is relatively significant .

It is possible that the tau-neutrino skims through a part of the ocean in addition to the 
Earth before exiting the interaction region 22]. Hence, it is desirable to compare the resulting tau-lepton fluxes as the tau neutrinos skim through mediums with different densities, while the slant depths of mediums are held fixed as an example. As stated before, the taulepton flux is essentially determined by the probability of $\nu_{\tau} N$ charged-current interaction happening within a tau-lepton range. Furthermore, from Fig. 1, it is clear that the taulepton range equals to the tau-lepton decay length for $E_{\tau}$ less than $10^{7} \mathrm{GeV}$. One therefore expects $F_{\tau}^{\text {rock }}(E, X) / F_{\tau}^{\text {water }}(E, X)=\rho^{\text {rock }} / \rho^{\text {water }}$ for $E_{\tau}<10^{7} \mathrm{GeV}$. This is clearly seen to be the case from Fig. 5 and Fig. 6, as we compare $F_{\tau}^{\text {rock }}$ with $F_{\tau}^{\text {water }}(E, X)$ for $X=2.65 \cdot 10^{6}$ $\mathrm{g} / \mathrm{cm}^{2}$ and $X=2.65 \cdot 10^{7} \mathrm{~g} / \mathrm{cm}^{2}$ respectively. For $E_{\tau}>10^{7} \mathrm{GeV}$, the tau-lepton range has additional dependencies on the mass density and the atomic number of the medium. Hence the ratio $F_{\tau}^{\text {rock }}(E, X) / F_{\tau}^{\text {water }}(E, X)$ starts deviating from $\rho^{\text {rock }} / \rho^{\text {water }}$. It is worthwhile to mention that the tau-lepton flux ratios for AGN and GRB cases behave rather similarly. On the other hand, the ratio in the GZK case has a clear peak between $10^{7.5}<E / \mathrm{GeV}<10^{8.5}$. Such a peak is even more apparent for the slant depth $X=2.65 \cdot 10^{7} \mathrm{~g} / \mathrm{cm}^{2}$. The appearance of this peak has to do with the relatively flat behavior of the incident GZK neutrino spectrum, while the position of this peak is related to the energy dependencies of the tau-lepton range and the neutrino-nucleon scattering cross sections. We have confirmed our observations by computing the flux ratios with simple power-law incident tau-neutrino fluxes. The above peak in the tau-lepton flux ratio implies the suppression of tau-lepton events from oceanskimming neutrinos compared to those from rock-skimming neutrinos. As stated earlier, the suppression of ocean-skimming neutrinos is related to the spectral index of the incident neutrino flux. It is therefore useful to perform a detailed simulation for it [23]. Such a detailed study is needed because the slant depths traversed by the above two kinds of neutrinos are generally different.

\section{PROSPECTS FOR POSSIBLE FUTURE OBSERVATIONS}

To observe the above tau leptons, the acceptance of a detector must be of the order of $\sim \mathrm{km}^{2}$ sr. For AGN neutrinos, the tau-lepton energy spectrum peaks at around $10^{7}$ to $10^{8}$ $\mathrm{GeV}$, which is below the threshold of a fluorescence detector, such as the High Resolution Fly's Eye (HiRes) 24]. Also, these tau leptons come near horizontally. At present, it seems

very difficult to construct a ground array in vertical direction. A Cherenkov telescope seems 
to be a feasible solution. In this context, NuTel collaboration is developing Cherenkov telescopes to detect the Earth-skimming high energy neutrinos [22]. However, because of the small opening angle of Cherenkov light cone and only a 10\% duty cycle (optical observations are limited to moonless and cloudless nights only), such a detector must cover very large area and field of view. A potential site for NuTel is at Hawaii Big Island, where two large volcanos, namely Mauna Loa and Mauna Kea, could be favorable candidates for high energy neutrinos to interact with. For a detector situated on top of Mount Hualalai and to look at both Mauna Kea and Mauna Loa, the required angular field of view is $\sim 8^{\circ} \times 120^{\circ}$. Furthermore, this telescope should have an acceptance area larger than $2 \mathrm{~km}^{2}$ sr so as to detect more than one event per year.

Concerning the GZK neutrinos, we note that the recent observation of ultra high energy cosmic rays by HiRes seem to be consistent with the GZK cutoff. Therefore a future observation of GZK tau neutrinos shall provide a firm support to GZK cutoff. In particular, the slight pile up of tau leptons between $10^{8} \mathrm{GeV}$ to $10^{9} \mathrm{GeV}$, induced by the Earth-skimming high energy GZK neutrinos, should be a candidate signature for GZK neutrinos. The integrated tau-lepton flux in this energy range is approximately $0.08 \mathrm{~km}^{-2} \mathrm{sr}^{-1} \mathrm{yr}^{-1}$. To detect one event per year from this flux, the acceptance of a detector must be larger than 120 $\mathrm{km}^{2} \mathrm{sr}$, for a fluorescence detector (assuming a duty cycle of 10\%). Although HiRes can reach $1000 \mathrm{~km}^{2}$ sr at energy $>3 \cdot 10^{9} \mathrm{GeV}$, it would be a technical challenge to lower down the threshold to $10^{8} \mathrm{GeV}$. Using a system similar to HiRes, the Dual Imaging Cherenkov Experiment (DICE) was able to detect Cherenkov light from extensive air-showers at energy as low as $10^{5} \mathrm{GeV}$ 255]. However, the field of view of DICE is also quite small, and thus several Cherenkov telescopes would be needed. An alternative method is a hybrid detection of both Cherenkov and fluorescence photons [26]. That is, a detector similar to HiRes, which looks at both land and sea and detects both Cherenkov and fluorescence photons, may observe the associated signal of GZK neutrinos.

In summary, we have given a semi-analytic treatment on the problem of simultaneous propagation of high energy tau neutrinos and tau leptons inside the Earth. Our treatment explicitly takes into account the inelasticity of neutrino-nucleon scatterings as well as the tau-lepton energy loss. We specifically considered the Earth-skimming situation and provided detailed results for the energy dependencies of emerging tau-lepton fluxes resulting from a few anticipated astrophysical neutrino fluxes. The effect of matter density on the 
tau-lepton flux is also studied. Such an effect is found to be related to the spectrum index of incident neutrino flux. Our treatment thus provides a basis for a more complete and realistic assessment of high-energy-neutrino flux measurements in the under-construction/planning large neutrino telescopes.

\section{Acknowledgements}

We thank N. La Barbera for communicating to us his Monte-Carlo-based results. HA thanks Physics Division of NCTS for support. MAH is supported by Taiwan's Ministry of Education under Research Excellence Project on Cosmology and Particle Astrophysics: Subproject II with the grant number 92-N-FA01-1-4-2. FFL, GLL, JJT and TWY are supported by National Science Council of Taiwan under the grant numbers NSC91-2112-M009-019 and NSC91-2112-M-001-024.

[1] S. W. Barwick, Astroparticle physics with high energy neutrinos, lectures presented at 28th SLAC Summer Institute on Particle Physics: Neutrinos from the Lab, the Sun, and the Cosmos (SSI 2000), Stanford, California, USA, 14-25 Aug 2000; H. Athar, arXiv:hep-ph/0209130, For a review article, see H. Athar, arXiv:hep-ph/0212387.

[2] G. Domokos and S. Kovesi-Domokos, arXiv:hep-ph/9801362; arXiv:hep-ph/9805221. See also, D. Fargion, Astrophys. J. 570, 909 (2002) arXiv:astro-ph/0002453.

[3] X. Bertou, P. Billoir, O. Deligny, C. Lachaud and A. Letessier-Selvon, Astropart. Phys. 17, 183 (2002) arXiv:astro-ph/0104452.

[4] J. L. Feng, P. Fisher, F. Wilczek and T. M. Yu, Phys. Rev. Lett. 88, 161102 (2002) arXiv:hep-ph/0105067.

[5] S. Bottai and S. Giurgola, Astropart. Phys. 18, 539 (2003) arXiv:astro-ph/0205325.

[6] L. V. Volkova, G. T. Zatsepin and L. A. Kuzmichev, Sov. J. Nucl. Phys. 29, 645 (1979) [Yad. Fiz. 29, 1252 (1979)].

[7] S. L. Glashow, Phys. Rev. 118, 316 (1960).

[8] For a recent discussion see, H. Athar and G. -L. Lin, Astropart. Phys. 19, 569 (2003) arXiv:hep-ph/0108204, and references cited therein. 
[9] S. I. Dutta, M. H. Reno, I. Sarcevic and D. Seckel, Phys. Rev. D 63, 094020 (2001) arXiv:hep-ph/0012350.

[10] B. Rossi, High Energy Particles (Prentice Hall, Englewood Cliffs, NJ, USA, 1952).

[11] A. A. Petrukhin and V. V. Shestakov, Can. J. Phys. 46, S377 (1968).

[12] R. P. Kokoulin and A. A. Petrukhin in Proceedings of the XII International Conference on Cosmic Rays (Hobart, Tasmania, Australia, 1971), Vol. 6.

[13] L. B. Bezrukov and E. V. Bugaev, Sov. J. Nucl. Phys. 33, 635 (1981) [Yad. Fiz. 33, 1195 (1981)]. For a recent discussion, see E. V. Bugaev and Y. V. Shlepin, Phys. Rev. D 67, 034027 (2003) arXiv:hep-ph/0203096.

[14] P. Lipari and T. Stanev, Phys. Rev. D 44, 3543 (1991).

[15] J. Pumplin, D. R. Stump, J. Huston, H. L. Lai, P. Nadolsky and W. K. Tung, JHEP 0207, 012 (2002) arXiv:hep-ph/0201195.

[16] V. A. Naumov and L. Perrone, Astropart. Phys. 10, 239 (1999) arXiv:hep-ph/9804301.

[17] A. Neronov, D. Semikoz, F. Aharonian and O. Kalashev, Phys. Rev. Lett. 89, 051101 (2002) arXiv:astro-ph/0201410; O. E. Kalashev, V. A. Kuzmin, D. V. Semikoz and G. Sigl, Phys. Rev. D 66,063004 (2002) arXiv:hep-ph/0205050.

[18] E. Waxman and J. N. Bahcall, Phys. Rev. Lett. 78, 2292 (1997) arXiv:astro-ph/9701231; ibid., Phys. Rev. D 59, 023002 (1999) arXiv:hep-ph/9807282.

[19] V. S. Berezinsky and G. T. Zatsepin, Phys. Lett. 28B, 423 (1969). For a recent discussion, see R. Engel, D. Seckel and T. Stanev, Phys. Rev. D 64, 093010 (2001) arXiv:astro-ph/0101216.

[20] H. Athar, arXiv:hep-ph/0210244 H. Athar, K. Cheung, G. -L. Lin and J. -J. Tseng, Astropart. Phys. 18, 581 (2003) arXiv:hep-ph/0112222.

[21] N. La Barbera, private communications.

[22] G. W. Hou and M. A. Huang, Expected performance of a neutrino telescope for seeing $A G N / G C$ behind a mountain, talk given in 1st NCTS Workshop on Astroparticle Physics, Taiwan, 6-9 Dec 2001, published in its proceedings, pp. 105-116, Eds. H. Athar, G. -L. Lin and K. -W. Ng, World Scientific, Singapore (2002) arXiv:astro-ph/0204145. See also http://hep1.phys.ntu.edu.tw/nutel/

[23] M. A. Huang, G. -L. Lin and J. -J. Tseng, work in progress.

[24] T. Abu-Zayyad et al. [HiRes-MIA Collaboration], Astrophys. J. 557, 686 (2001) arXiv:astro-ph/0010652. 
[25] S. P. Swordy and D. B. Kieda, Astropart. Phys. 13, 137 (2000) arXiv:astro-ph/9909381.

[26] Z. Cao, M. A. Huang, P. Sokolsky, and R. W. Springer, Proc. of the 28th Int'l Cosmic Ray Conf., Tsukuba, Japan, July-August 2003, in preparation. 


\section{APPENDIX A: THE CONTRIBUTION FROM RESONANT $\bar{\nu}_{e} e^{-}$SCATTERING}

The transport equations for $\bar{\nu}_{e}$ and the tau lepton are given by Eqs. (3) and (44). For convenience, let us write $1-y=z$. The last term in Eq. (41) can be simplified using

$$
\frac{\mathrm{d} \sigma_{\bar{\nu}_{e} e^{-} \rightarrow \bar{\nu}_{\tau} \tau^{-}}}{\mathrm{d} z}(z, E / z)=\frac{m_{W}^{4} G_{F}^{2}}{\pi} \frac{s(1-z)^{2}}{\left(s-m_{W}^{2}\right)^{2}+m_{W}^{2} \Gamma_{W}^{2}},
$$

and the narrow-width approximation

$$
\frac{1}{\pi} \frac{m_{W} \Gamma_{W}}{\left(s-m_{W}^{2}\right)^{2}+m_{W}^{2} \Gamma_{W}^{2}} \approx \delta\left(s-m_{W}^{2}\right)
$$

We arrive at

$$
\frac{\partial F_{\tau}(E, X)}{\partial X}=-\frac{F_{\tau}(E, X)}{\rho d_{\tau}(E)}+\frac{1}{3}\left(1-\frac{E}{E_{R}}\right)^{2}\left(\frac{\pi \Gamma_{W}}{L_{R} m_{W}}\right) F_{\bar{\nu}_{e}}\left(E_{R}, X\right),
$$

where $E_{R}=m_{W}^{2} / 2 m_{e}$ is the $\bar{\nu}_{e}$ energy such that the $W$ boson is produced on-shell in the $\bar{\nu}_{e} e^{-}$scattering. The $L_{R} \equiv 1 / n_{e} \sigma_{\bar{\nu}_{e} e^{-} \rightarrow W^{-}}$is the interaction thickness for such a scattering. To solve for $F_{\tau}(E, X)$, we need to input $F_{\bar{\nu}_{e}}\left(E_{R}, X\right)$. Obviously, the $\bar{\nu}_{e}$ flux at the

resonant-scattering energy $E_{R}$ is mainly attenuated by the resonant scattering itself. Hence $F_{\bar{\nu}_{e}}\left(E_{R}, X\right)=\exp \left(-X / L_{R}\right) F_{\bar{\nu}_{e}}\left(E_{R}, 0\right)$. Substituting this result into Eq. (A3), we obtain

$$
\begin{aligned}
F_{\tau}(E, X) & =\frac{1}{3}\left(1-\frac{E}{E_{R}}\right)^{2}\left(\frac{\pi \Gamma_{W}}{L_{R} m_{W}}\right) F_{\bar{\nu}_{e}}\left(E_{R}, 0\right) \exp \left(-\frac{X}{\rho d_{\tau}(E)}\right) \\
& \times \int_{0}^{X} \mathrm{~d} Z \exp \left[\left(\frac{1}{\rho d_{\tau}(E)}-\frac{1}{L_{R}}\right) Z\right]
\end{aligned}
$$

The integration over $Z$ can be easily performed. In practice, it is obvious that $X \gg \rho d_{\tau}(E)$. In this limit, we have

$$
F_{\tau}(E, X)=\frac{\pi}{3}\left(1-\frac{E}{E_{R}}\right)^{2}\left(\frac{\Gamma_{W}}{m_{W}}\right)\left(\frac{\rho d_{\tau}(E)}{L_{R}}\right) F_{\bar{\nu}_{e}}\left(E_{R}, 0\right) \exp \left(-\frac{X}{L_{R}}\right) .
$$

Let us consider standard rock as the medium for $\bar{\nu}_{e} e^{-}$scattering, we then have $\rho / L_{R}=$ $n_{e} \rho \sigma_{\bar{\nu}_{e} e^{-} \rightarrow W^{-}}$. Given $\sigma_{\bar{\nu}_{e} e^{-} \rightarrow W^{-}}=4.8 \cdot 10^{-31} \mathrm{~cm}^{2}$ at the $\mathrm{W}$ boson mass peak, and $n_{e} \rho=$ $2.65 \times 6.0 / 2 \times 10^{23} / \mathrm{cm}^{3}$ in the standard rock, we obtain $\rho / L_{R}=(26 \mathrm{~km})^{-1}$. Furthermore, we can write $d_{\tau}(E)=49 \mathrm{~km} \times\left(E / 10^{6} \mathrm{GeV}\right)$. We then obtain the following ratio

$$
\frac{F_{\tau}(E, X)}{F_{\bar{\nu}_{e}}\left(E_{R}, 0\right)}=3.3 \cdot 10^{-4} \times\left(\frac{E}{E_{R}}\right) \times\left(1-\frac{E}{E_{R}}\right)^{2} \times \exp \left(-\frac{X}{L_{R}}\right) .
$$

This is the result given by Eq. (10) in the main text. 
APPENDIX B: THE ITERATION METHOD FOR OBTAINING THE $Z_{\nu}(E, X)$ AND THE $F_{\tau}(E, X)$

The evolution for $F_{\nu_{\tau}}$ is given by Eq. (11). With the ansatz

$$
F_{\nu_{\tau}}(E, X)=F_{\nu_{\tau}}(E, 0) \exp \left(-\frac{X}{\Lambda_{\nu}(E, X)}\right),
$$

we obtain the following equation for $Z_{\nu}(E, X)$ :

$$
\begin{aligned}
X Z_{\nu}(E, X) & =\int_{0}^{X} \mathrm{~d} X^{\prime} \int_{0}^{1} \frac{\mathrm{d} y}{1-y}\left\{\frac{F_{\nu_{\tau}}^{(0)}\left(E_{y}\right)}{F_{\nu_{\tau}}^{(0)}(E)} \exp \left[-X^{\prime} D_{\nu}\left(E, E_{y}, X^{\prime}\right)\right] \Phi_{\nu_{\tau}}^{\mathrm{NC}}(y, E)\right. \\
& +\frac{F_{\tau}\left(E_{y}, X^{\prime}\right)}{F_{\nu_{\tau}}^{(0)}(E)}\left(\frac{\lambda_{\nu}(E)}{\rho d_{\tau}(E)}\right) \exp \left(\frac{X^{\prime}}{\Lambda_{\nu}\left(E, X^{\prime}\right)}\right) \Phi_{\tau}^{d}(y, E) \\
& \left.+\frac{F_{\tau}\left(E_{y}, X^{\prime}\right)}{F_{\nu_{\tau}}^{(0)}(E)}\left(\frac{\lambda_{\nu}(E)}{\lambda_{\tau}(E)}\right) \exp \left(\frac{X^{\prime}}{\Lambda_{\nu}\left(E, X^{\prime}\right)}\right) \Phi_{\tau}^{\mathrm{CC}}(y, E)\right\},
\end{aligned}
$$

where $F_{\nu_{\tau}}^{(0)}(E) \equiv F_{\nu_{\tau}}(E, 0)$, while $\Phi_{\nu_{\tau}}^{\mathrm{NC}}, \Phi_{\tau}^{\mathrm{CC}}$ and $\Phi_{\tau}^{d}$ are respectively given by

$$
\begin{aligned}
\Phi_{\nu_{\tau}}^{\mathrm{NC}}(y, E) & =\frac{\sum_{T} n_{T} \frac{\mathrm{d} \sigma_{\nu_{\tau} T \rightarrow \nu_{\tau} Y}}{\mathrm{~d} y}\left(y, E_{y}\right)}{\sum_{T} n_{T} \sigma_{\nu_{\tau} T}^{\text {tot }}(E)}, \\
\Phi_{\tau}^{\mathrm{CC}}(y, E) & =\frac{\sum_{T} n_{T} \frac{\mathrm{d} \sigma_{\tau T \rightarrow \nu_{\tau} Y}}{\mathrm{~d} y}\left(y, E_{y}\right)}{\sum_{T} n_{T} \sigma_{\tau T}^{\text {tot }}(E)}, \\
\Phi_{\tau}^{d}(y, E) & =\frac{1}{\Gamma_{\tau}(E)} \frac{\mathrm{d} \Gamma_{\tau \rightarrow \nu_{\tau} Y}}{\mathrm{~d} y}\left(y, E_{y}\right),
\end{aligned}
$$

with $n_{T}$ the number of targets per unit mass of the medium, and

$$
D_{\nu}\left(E, E_{y}, X\right)=\frac{1}{\Lambda_{\nu}\left(E_{y}, X\right)}-\frac{1}{\Lambda_{\nu}(E, X)}
$$

For the simplicity in notations, we take the lower and upper limits for the $y$ integration to be 0 and 1 respectively. In reality, the limits depend on the actual kinematics of each process. One may impose these limits in the functions $\Phi_{\nu_{\tau}}^{\mathrm{NC}}, \Phi_{\tau}^{\mathrm{CC}}$ and $\Phi_{\tau}^{d}$.

To perform the iteration, we begin by setting $Z_{\nu(0)}=0$. In this approximation, we have

$$
F_{\nu_{\tau}(0)}(E, X)=F_{\nu_{\tau}}(E, 0) \exp \left(-\frac{X}{\lambda_{\nu}(E, X)}\right) \text {. }
$$


Substituting $F_{\nu_{\tau}(0)}(E, X)$ into Eq. (12), we obtain the lowest order $\nu_{\tau}$ flux, $F_{\tau(0)}(E, X)$. The first iteration for $Z_{\nu}$, denoted by $Z_{\nu(1)}$ is calculable from Eq. (B2) by substituting $F_{\nu_{\tau}(0)}(E, X), F_{\tau(0)}(E, X)$, and $Z_{\nu(0)}$ into the R.H.S. of this equation. From $Z_{\nu(1)}$, we can then calculate $F_{\nu_{\tau}(1)}(E, X)$ and $F_{\tau(1)}(E, X)$, which corresponds to the results presented in this paper. We have checked the convergence of iteration procedure and have found negligible differences between $Z_{\nu(2)}$ and $Z_{\nu(1)}$ and their associated $\nu_{\tau}$ and $\tau$ fluxes.

The value of $Z_{\nu}$ depends on the spectrum index of the neutrino flux, since it effectively gives the regeneration effect in the neutrino-nucleon scattering. In general, a flatter neutrino spectrum implies a larger $Z_{\nu}$. The $Z_{\nu}$ is however not sensitive to the slant depth $X$. In the case of GRB neutrinos, where the flux decreases as $E_{\nu}^{-2}$ for $E_{\nu}<10^{7} \mathrm{GeV}$, and decreases as $E_{\nu}^{-3}$ for energies greater than that, we obtain $Z_{\nu}^{\mathrm{GRB}} \approx 0.2$. For the AGN neutrino, $Z_{\nu}^{\mathrm{AGN}}$ changes from 0.96 to 0.35 as $E_{\nu}$ runs from $10^{5} \mathrm{GeV}$ to $10^{6} \mathrm{GeV}$. In this energy range, the neutrino flux decreases slower than $E_{\nu}^{-0.5}$. For $E_{\nu}$ greater than $10^{8} \mathrm{GeV}, Z_{\nu}^{\text {AGN }}$ drops below 0.2 as the neutrino flux spectrum begins a steep fall. The values for $Z_{\nu}^{\mathrm{GZK}}$ also follow the similar pattern. 
TABLE I: Comparison of the integrated tau-lepton flux $\left(\mathrm{km}^{-2} \mathrm{yr}^{-1} \mathrm{sr}^{-1}\right)$ in different energy bins for the AGN, the GRB and the GZK neutrinos without and with approximation (see text for details). The distance traversed is taken to be $10 \mathrm{~km}$ in rock here. For $10^{9} \leq E / \mathrm{GeV} \leq 10^{10}$, the incident AGN neutrino flux is too small so that its induced tau-lepton flux is not shown.

\begin{tabular}{|c|c|c|c|c|c|c|}
\hline \hline \multirow{2}{*}{ Energy Interval } & \multicolumn{2}{|c|}{$A G N$} & \multicolumn{2}{c|}{$G R B$} & \multicolumn{2}{c|}{$G Z K$} \\
\cline { 2 - 7 } & full & approx & full & approx & full & approx \\
\hline $10^{6} \leq E / \mathrm{GeV} \leq 10^{7}$ & 2.23 & 2.12 & $9.63 \cdot 10^{-3}$ & $1.05 \cdot 10^{-2}$ & $7.38 \cdot 10^{-5}$ & $2.08 \cdot 10^{-5}$ \\
$10^{7} \leq E / \mathrm{GeV} \leq 10^{8}$ & 4.89 & 5.12 & $7.12 \cdot 10^{-3}$ & $6.82 \cdot 10^{-3}$ & $1.14 \cdot 10^{-2}$ & $1.90 \cdot 10^{-2}$ \\
$10^{8} \leq E / \mathrm{GeV} \leq 10^{9}$ & $1.95 \cdot 10^{-1}$ & $1.52 \cdot 10^{-1}$ & $5.39 \cdot 10^{-4}$ & $4.63 \cdot 10^{-4}$ & $8.17 \cdot 10^{-2}$ & $8.47 \cdot 10^{-2}$ \\
$10^{9} \leq E / \mathrm{GeV} \leq 10^{10}$ & & & $1.13 \cdot 10^{-5}$ & $1.24 \cdot 10^{-5}$ & $3.31 \cdot 10^{-2}$ & $3.52 \cdot 10^{-2}$ \\
\hline \hline
\end{tabular}




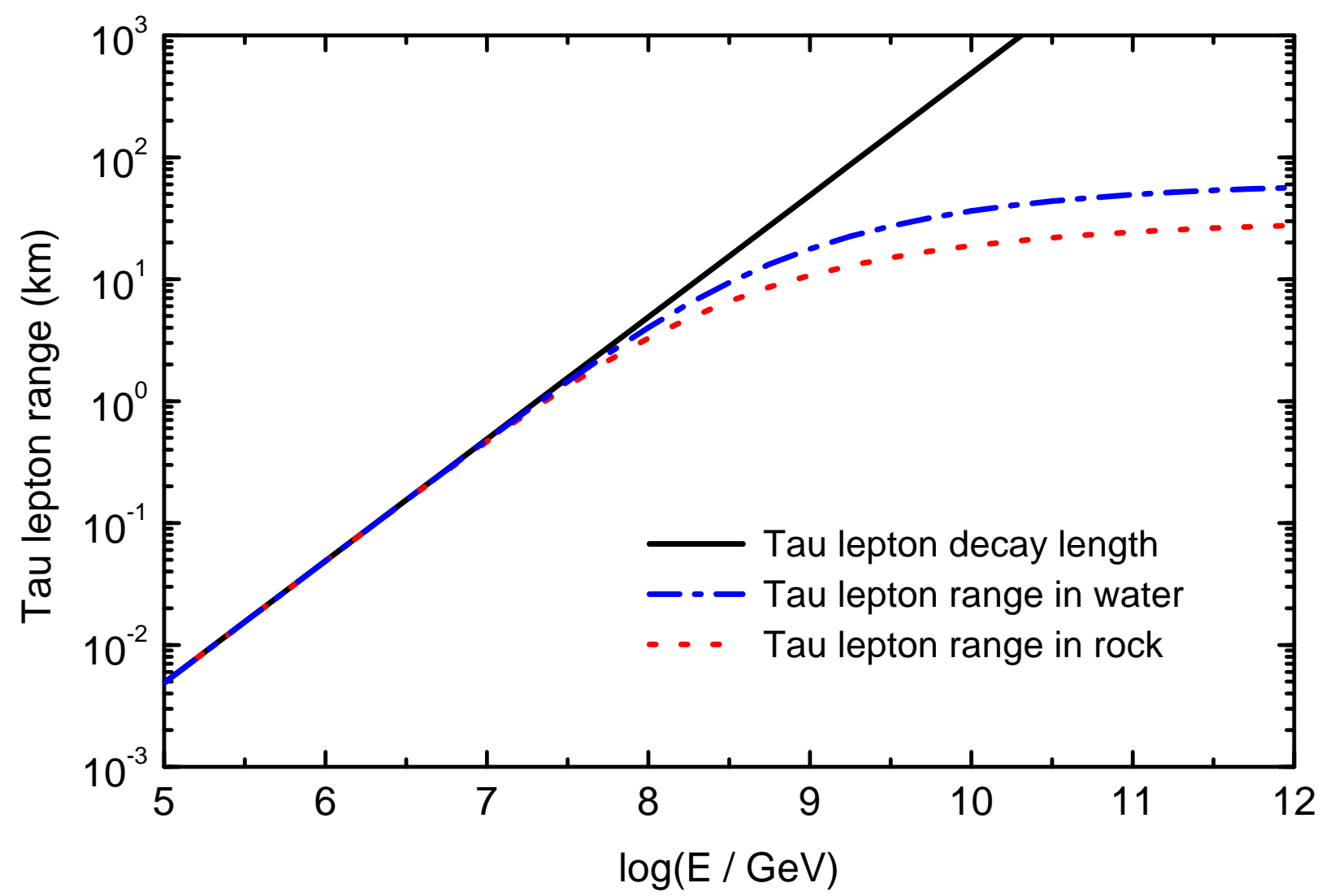

FIG. 1: The tau-lepton range in rock and in water using Eq. 8 and the tau-lepton decay length $d_{\tau}$ in $\mathrm{km}$ as a function of tau-lepton energy in $\mathrm{GeV}$. 


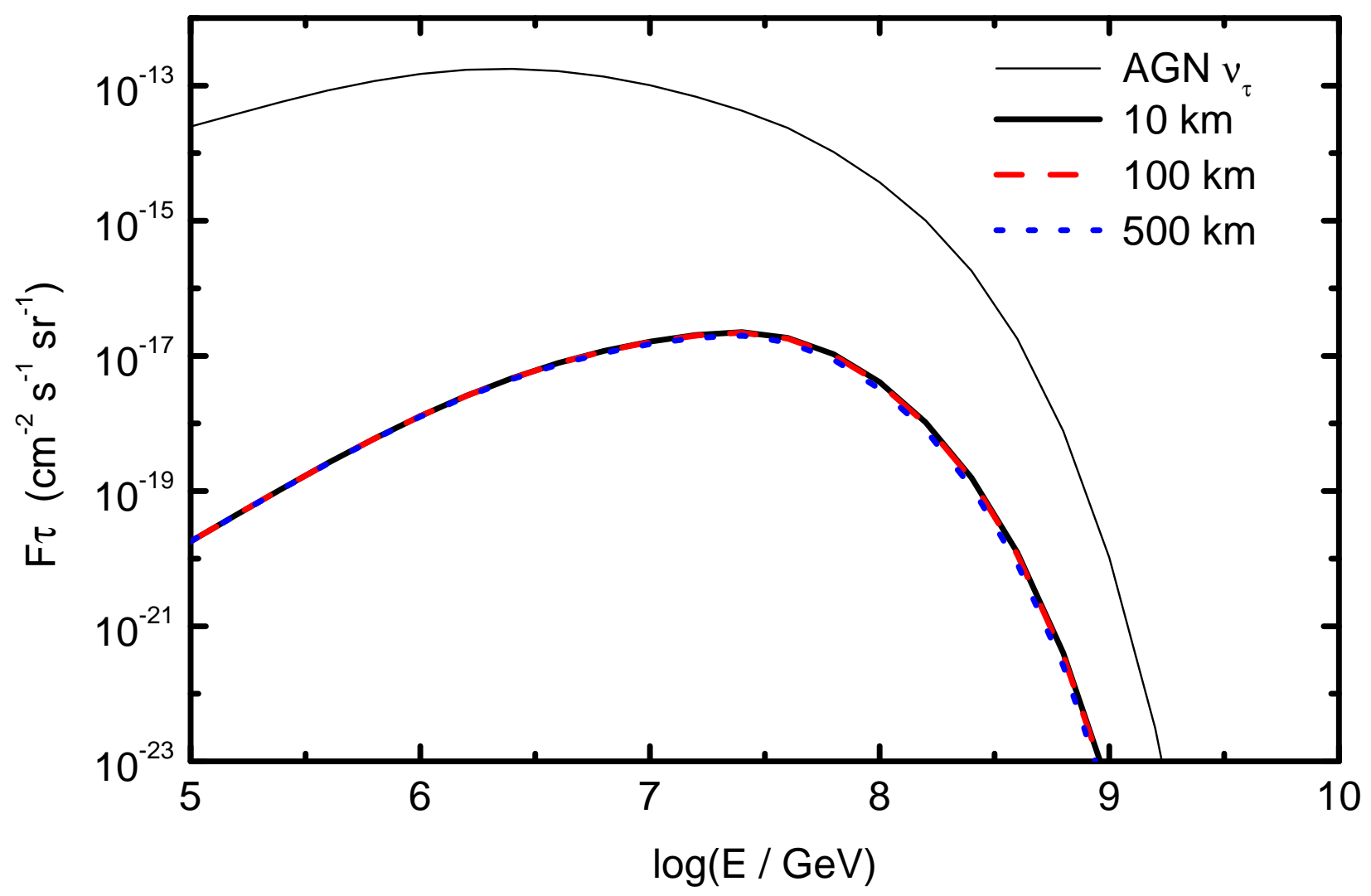

FIG. 2: The tau-lepton energy spectrum induced by the AGN neutrinos in rock for three different $X / \rho$ ratio values (see text for more details). The incident tau-neutrino flux is shown by the thin solid line. 


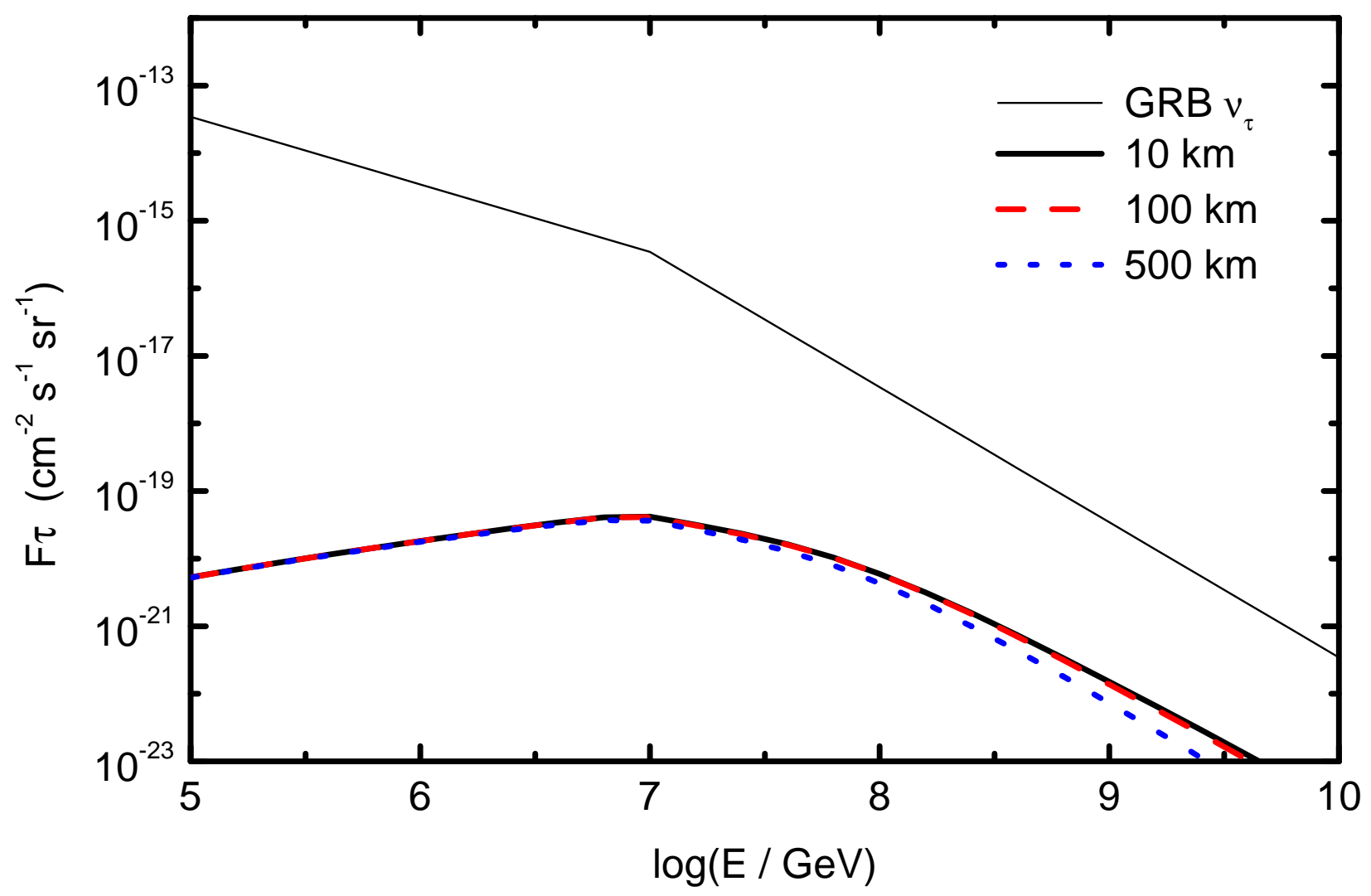

FIG. 3: The tau-lepton energy spectrum induced by the GRB neutrinos in rock for three different $X / \rho$ ratio values (see text for more details). The incident tau-neutrino flux is shown by the thin solid line. 


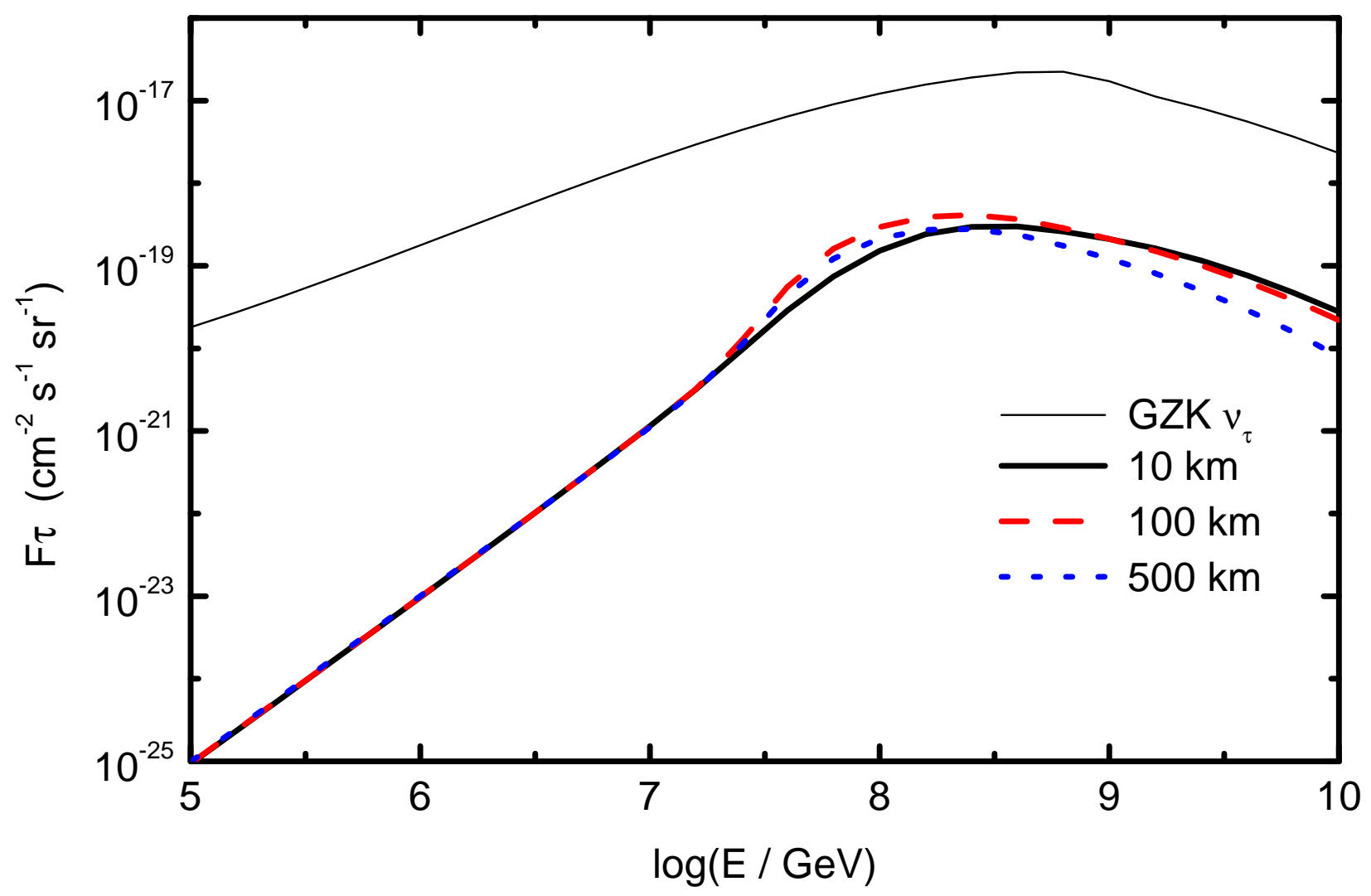

FIG. 4: The tau-lepton energy spectrum induced by the GZK neutrinos in rock for three different $X / \rho$ ratio values (see text for more details). The incident tau-neutrino flux is shown by the thin solid line. 


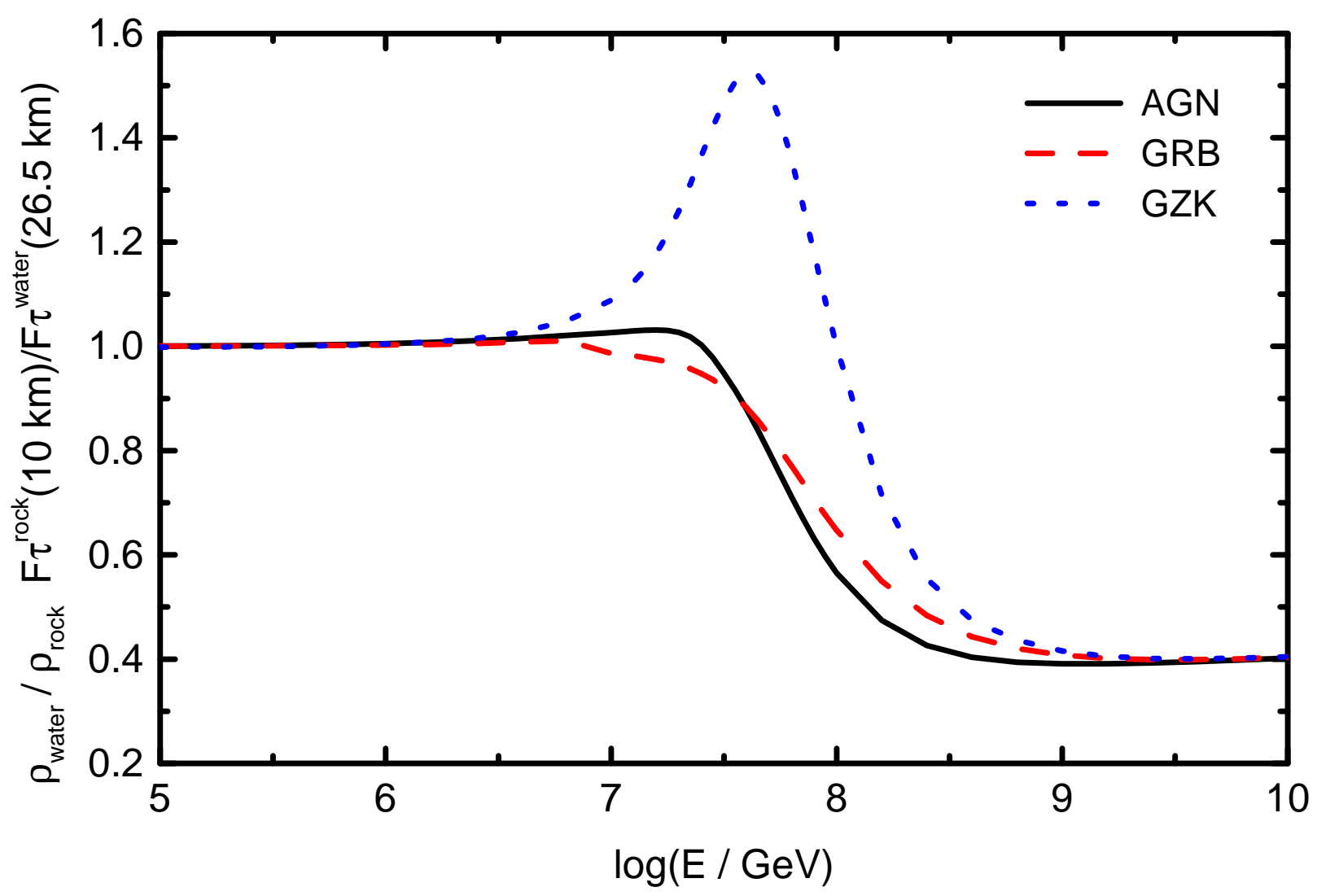

FIG. 5: The ratio of $F_{\tau}$ in rock and water induced by the AGN, the GRB and the GZK neutrinos for $X=2.65 \cdot 10^{6} \mathrm{~g} / \mathrm{cm}^{2}$. 


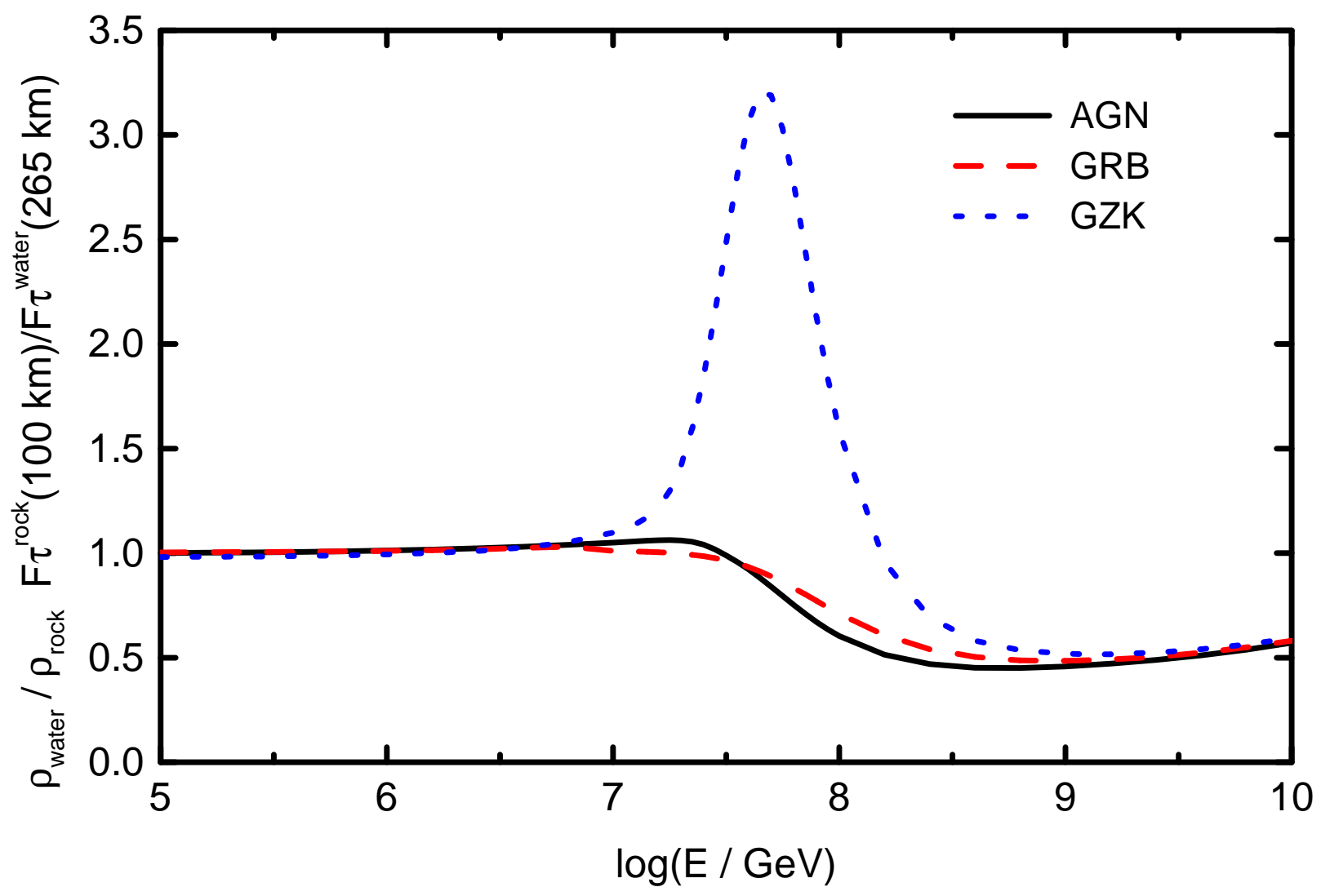

FIG. 6: The ratio of $F_{\tau}$ in rock and water induced by the AGN, the GRB and the GZK neutrinos for $X=2.65 \cdot 10^{7} \mathrm{~g} / \mathrm{cm}^{2}$. 